\title{
Unusual Type of Hand Preaxial Polydactyly
}

\author{
Srinivasa Rao Sirasanagandla ${ }^{1}$ Satheesha B. Nayak ${ }^{2}$ Mohamed Al Mushaiqri ${ }^{1}$ \\ ${ }^{1}$ Department of Human and Clinical Anatomy, College of Medicine \\ and Health Sciences, Sultan Qaboos University, Muscat, Oman \\ 2 Department of Anatomy, Melaka Manipal Medical College (Manipal \\ Campus), Manipal Academy of Higher Education, Manipal, \\ Address for correspondence Satheesha B. Nayak, PhD, Department \\ of Anatomy, Melaka Manipal Medical College (Manipal Campus), \\ Manipal Academy of Higher Education, Madav Nagar, Manipal, \\ Karnataka, India. 567104 (e-mail: nayaksathish@gmail.com).
} Karnataka State, India

J Morphol Sci 2018;35:170-172.

Abstract
Keywords
- radial polydactyly
- congenital anomalies
- cadaver
- hand

Preaxial polydactyly is a common congenital anomaly of the hand. In this anomaly, an additional digit is seen along the preaxial border of the thumb. Several possible types of preaxial polydactyly have been observed and categorized into several types based on the level of duplications. In the present case, we report an unusual type of polydactyly in an approximately 55year-old male cadaver of South Indian origin. An additional nonfunctional digit was noted along the preaxial border of the thumb, at the level of the base of the distal phalanx. Upon careful dissection of the anomalous digit, a thick cord of connective tissue formed by the fibrous flexor sheath around the flexor pollicis longus tendon was noted in its central core. This cord was surrounded by a mass of fatty tissue. The skeletal, muscle and tendon components were found to be absent in the additional digit.

\section{Introduction}

Polydactyly is one of the frequently observed congenital anomalies of the hand and foot. In this anomaly, a rudimentary or fully developed additional digit is seen along with the usual digits. The anomaly is also referred as polydactylia, polydactylism, or hyperdactyly. ${ }^{1}$ It can occur as a part of a syndrome or isolatedly. ${ }^{2}$ Polydactyly of the hand or of the foot are categorized into preaxial, postaxial and central types. ${ }^{3}$ Thumb polydactyly is the most common type of polydactyly in the hand. Currently, the classification of thumb polydactyly proposed by Wassel ${ }^{4}$ is in clinical use. In the present case, we report an unusual type of preaxial polydactyly and discuss its structural components.

\section{Case Report}

During regular dissections for medical graduates, we came across a rare type of preaxial polydactyly in the left hand. It was observed in an $\sim 55$-year-old formalin embalmed male cadaver of South Indian origin. The additional digit was present on the

received

March 24, 2017

accepted

September 6, 2018

published online

November 6, 2018
DOI https://doi.org/

10.1055/s-0038-1675571. ISSN 2177-0298. lateral margin (preaxial border) of the thumb. The extra digit was $\sim 1.4 \mathrm{~cm}$ in length, and it was found to be budding at the level of the base of the distal phalanx of the thumb ( - Fig. 1 and 2). A dissection was performed to evaluate the structural framework of the digit. Upon the reflection of the skin, a mass of fatty tissue was observed ( - Fig. 3). Upon further removal of the fatty tissue, a thick cord of connective tissue was noted in its central core. This cord of connective tissue was found to be continues with the fibrous flexor sheath around the flexor pollicis longus tendon ( - Fig. 3 and $\mathbf{4}$ ). The additional digit was non-functional as there were no bone, muscle or tendon components in it (-Fig. $\mathbf{3}$ and $\mathbf{4}$ ).

\section{Discussion}

The pathogenesis of preaxial polydactyly has been studied in both experimental animals ${ }^{5,6}$ and in men. ${ }^{7}$ Yasuda has studied 13 human embryos and demonstrated the morphological features in the early pathogenesis of preaxial polydactyly. ${ }^{7}$ In this study, it is concluded that the interaction between the ectoderm and the mesoderm of the limb plays a crucial role in the development of preaxial

Copyright @ 2018 by Thieme Revinter Publicações Ltda, Rio de Janeiro, Brazil
License terms

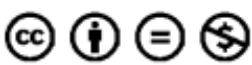




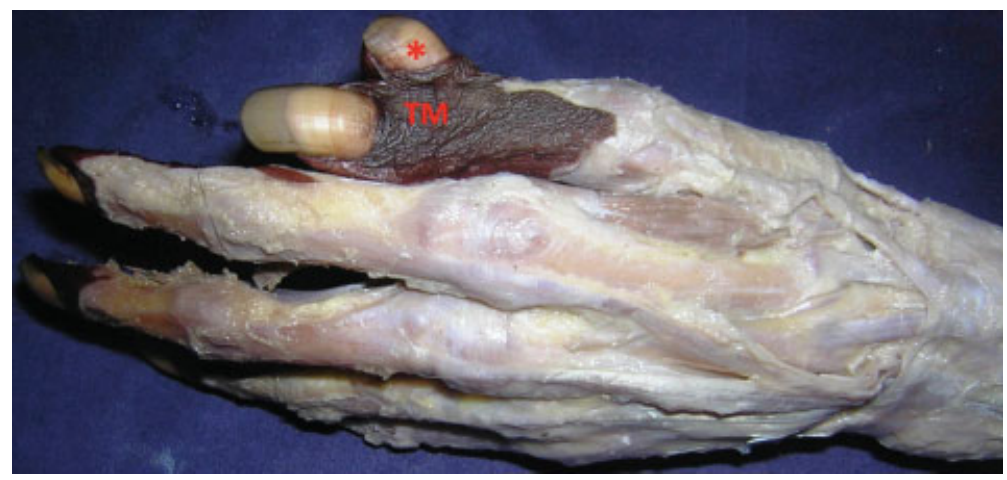

Fig. 1 Photograph of the dorsal view of the left hand showing the thumb polydactyly. Note the additional digit (asterisk) along the preaxial border of the thumb (TM).

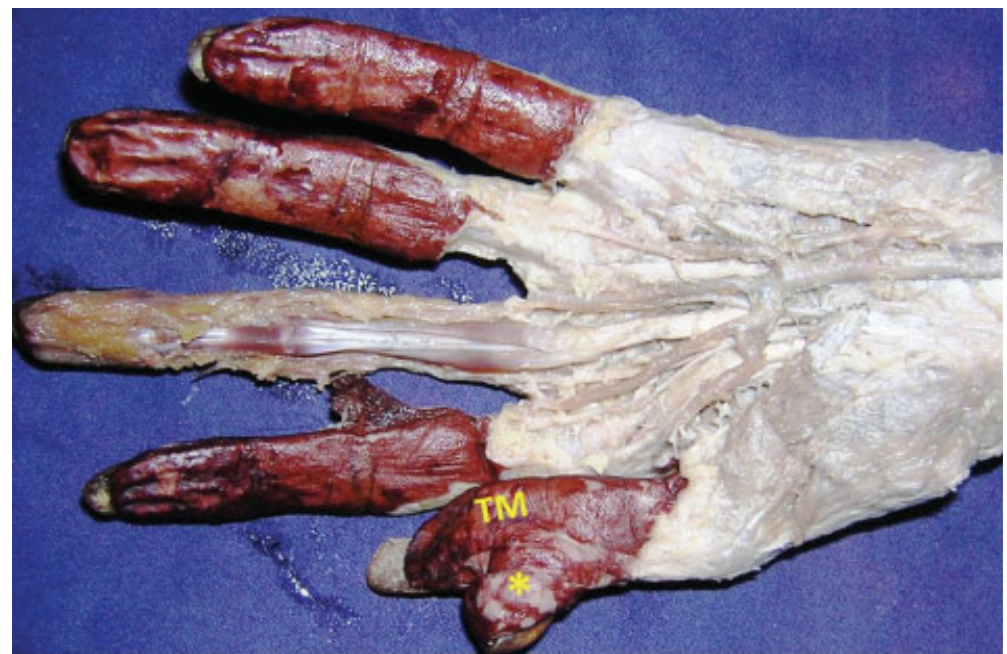

Fig. 2 Photograph of the palmar view of the left hand showing the thumb polydactyly. Note the additional digit (asterisk) along the preaxial border of the thumb (TM).

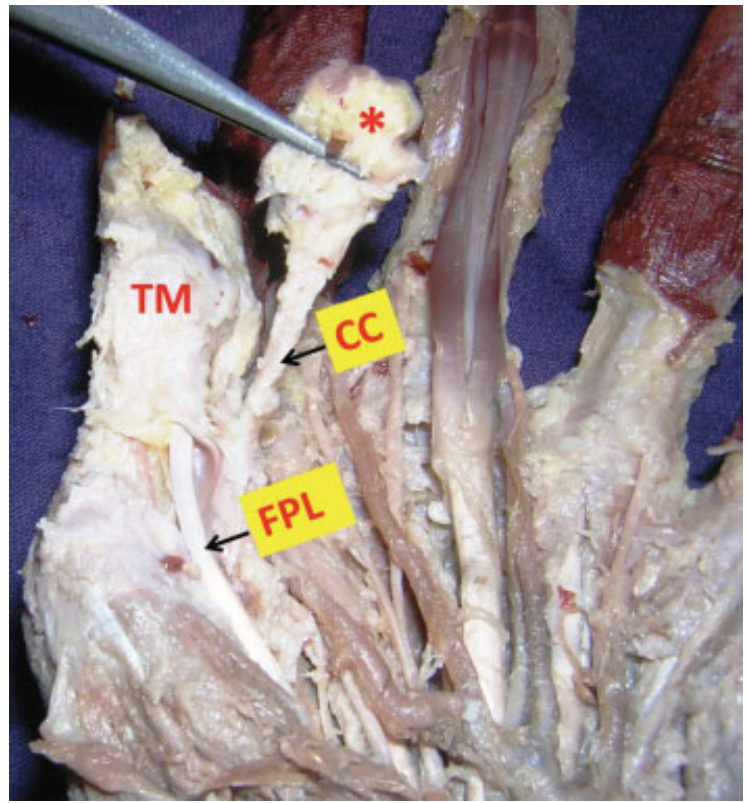

Fig. 3 Dissection of the additional digit (asterisk) showing the connective tissue cord (CC) in its central core. Note the formation of the CC from the fibrous flexor sheath around the flexor pollicis longus tendon (FPL). (TM: thumb). polydactyly. ${ }^{7}$ In both white and black populations, the incidence of thumb polydactyly is found to occur in 8 in 100,000 individuals. $^{8,9}$ The Wassel classification ${ }^{4}$ is proposed based on the level of bifurcation or duplication, and is widely accepted clinically. According to Wassel, the pathoanatomy of thumb polydactyly can be divided into 7 groups; type I: bifid distal phalanx; type II: duplicated distal phalanx; type III: bifid proximal phalanx; type IV: duplication of the proximal phalanx that rests on the broad metacarpal; type V: bifid metacarpal; type VI: duplicated metacarpal; and type VII: triphalangism. ${ }^{4}$ The occurrence of type IV is more common when compared with other types. ${ }^{4}$ In the present case, we report an unusual type of thumb polydactyly in which the additional thumb was found to be devoid of skeletal, muscle and tendon components. In the Wassel ${ }^{4}$ classification of thumb polydactyly, the reported type has not been described. The documentation of this rare type may be clinically important. In an immature hand, due to the presence of the cartilaginous epiphysis, the identification of the level of bifurcation between the duplicated components is difficult. ${ }^{3}$ This can possibly explain why sometimes the clinically diagnosed type of polydactyly is different from the findings 


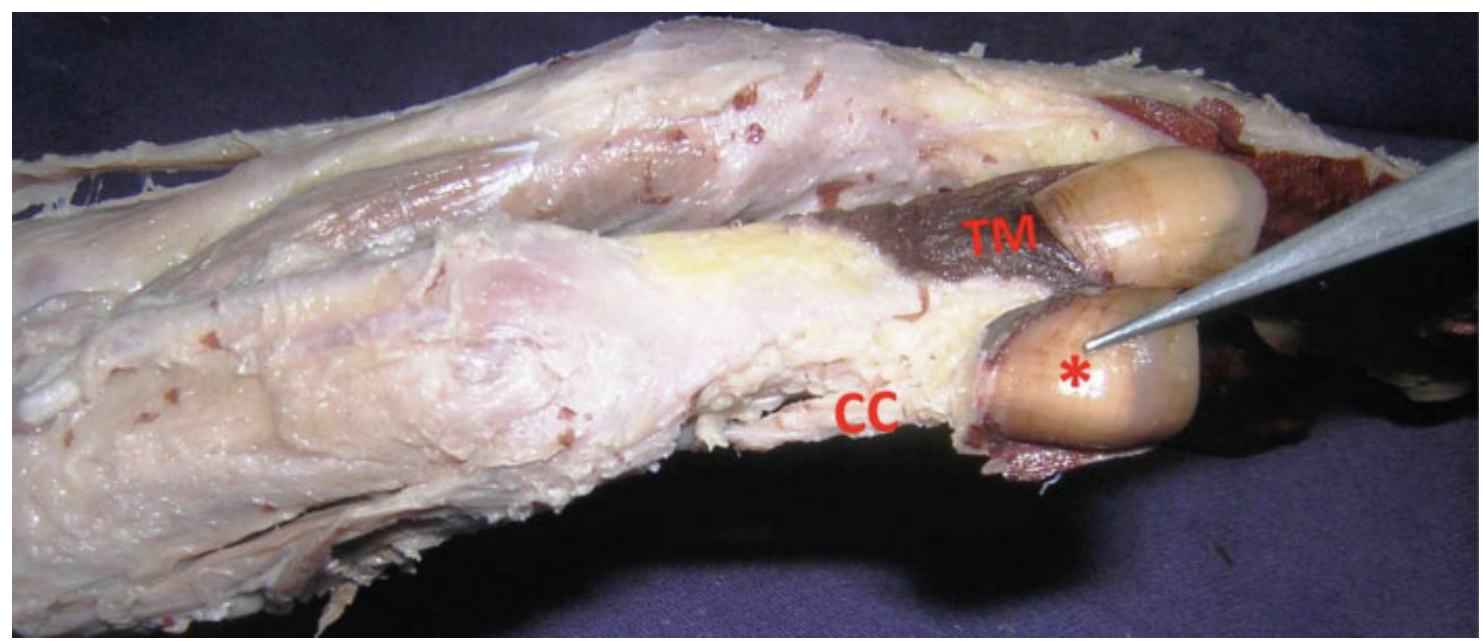

Fig. 4 Photograph of the dorsal view of the left hand showing the additional digit (asterisk) connected to the normal digit (TM: thumb) by the connective tissue cord (CC).

encountered in the surgery and, in such cases, the classification may not be appropriate to deal with. ${ }^{3}$ A radiographic examination is usually recommended to evaluate the presence of the skeletal component in the additional digit, before planning the surgery. ${ }^{3}$ For the better reconstruction outcome and to avoid or reduce complications in operations, the components of the additional digit such as skin, nail, bone, and the ligaments should be evaluated and treated in a simultaneous manner. ${ }^{9,10}$ The additional digit observed in the present case was non-functional and there were no skeletal, muscle and tendon components in it. The presence of a thick cord of connective tissue formed by the fibrous flexor sheath of the flexor pollicis longus tendon reported here may be clinically significant during the diagnosis, treatment and while planning the surgery and reconstruction procedures.

\section{Conflicts of Interest}

The authors have no conflicts of interest to declare.

\section{References}

1 Zayed EF, Elbanoby TM, Ayad W, El-Shishtawy AM. Polydactyly: Surgical Strategy and Clinical Experience with 40 Cases. Egypt J Plast Reconstr Surg 2011;35:287-300

2 Blauth W, Olason AT. Classification of polydactyly of the hands and feet. Arch Orthop Trauma Surg 1988;107(06):334-344

3 Mumoli N, Gandini D, Wamala EK, Cei M. Left hand polydactyly: a case report. Cases J 2008;1(01):346

4 Wassel HD. The results of surgery for polydactyly of the thumb. A review. Clin Orthop Relat Res 1969;64(64):175-193

5 Zwtlling E. Abnormal morphogenesis in limb development. In Limb Development and Deformity: Problems of Evaluation and Rehabilitation (ed. C. A. Swinyard), Springfield: C. C. Thomas: 1969.pg.100-113.

6 Kameyama Y, Hayashi I, Hoshino K. Morphogenesis of 5-fiuorouracil induced polydactylism in mice. Teratology 1973;8:95-96

7 Yasuda M. Pathogenesis of preaxial polydactyly of the hand in human embryos. J Embryol Exp Morphol 1975;33(03):745-756

8 Ezaki M. Radial polydactyly. Hand Clin 1990;6(04):577-588

9 Graham TJ, Ress AM. Finger polydactyly. Hand Clin 1998;14(01): 49-64

10 Netscher DT, Baumholtz MA. Treatment of congenital upper extremity problems. Plast Reconstr Surg 2007;119(05):101e-129e 